\title{
MODELO DE PLANEACIÓN Y PROGRAMACIÓN DE LA PRODUCCIÓN PARA EL TROQUELADO DE CUERO EN LA INDUSTRIA DE CALZADO
}

\section{MODEL OF PLANNING AND PROGRAMMING OF THE PRODUCTION FOR THE CUTTING OF LEATHER IN THE FOOTWEAR INDUSTRY}

\author{
John Paul Reyes Vásquez", , Israel Altamirano Zanipatin', Darwin Santiago Aldás \\ Salazar ${ }^{1}$, Luis Alberto Morales Perrazo, Claudio Renato Reyes Vásquez ${ }^{2}$
}

\begin{abstract}
RESUMEN
Se propone desarrollar un modelo de programación de la producción en el proceso de troquelado de calzado, con el fin de generar una carga uniforme de trabajo. Inicialmente se identifican las restricciones que limitan el proceso empleando la Teoría de Restricciones y la herramienta TAC (Tambor-Amortiguador-Cuerda). El cálculo de la capacidad de producción define el cuello de botella que marca el ritmo de producción (tambor). El modelo de programación (Cuerda) se basa en un sistema de producción Pull de Lean Manufacturing para sincronizar la manufactura del calzado con los procesos de aparado y montaje de acuerdo con la filosofía Justo a Tiempo (en inglés JIT). Con células de manufactura se logra optimizar eficiente la maquinaria y el recurso humano (amortiguador). Los resultados del modelo radican en estimar el número de turnos necesarios para cumplir con los requerimientos de producción. Comparando el modelo actual de producción con el propuesto se tiene un aumento que va desde el $10 \%$ hasta el $27 \%$ de eficiencia en la capacidad de producción.
\end{abstract}

Palabras Clave: Restricciones de proceso, programación de producción, Sistema Pull

\section{ABSTRACT}

It is proposed to develop a model for programming production in the cutting process, to generate a uniform workload. Initially, the restrictions that limit the process are identified using the Theory of Constraints and the Drum-Buffer-Rope tool. The calculation of the production capacity defines the bottleneck that marks the rhythm of production (drum). The programming

\footnotetext{
1 Universidad Técnica de Ambato. Ambato, Ecuador. orcid.org/0000-0002-5446-5490; orcid.org/0000-0003-0026-8042; orcid.org/0000-0001-8882-030X; orcid.org/0000-0002-0921-262X

2Universidad Técnica Particular de Loja. Loja, Ecuador. orcid.org/0000-0001-8332-258X
} 
model (Rope) is based on a production system Pull of Lean Manufacturing to synchronize the manufacture of footwear with the processes of stitching and assembly according to the Just in Time (JIT) philosophy. With manufacturing cells, it is possible to optimize machinery and human resources (Buffer) efficiently. The model results show the number of shifts required to meet production requirements. This compares the current production model with the one proposed, and there is an increase ranging from $10 \%$ to $27 \%$ efficiency in production capacity.

Keywords: Process Constraints, production scheduling, System Pull

\section{INTRODUCCIÓN}

La globalización ha cambiado el mundo, así como la forma en que un cliente juzga el valor de un producto, lo que ha creado un nuevo enfoque de negocio. En esta dirección se han orientado diferentes enfoques, como el sistema de manufactura esbelta, se acepta generalmente que la producción esbelta mejora los procesos de fabricación a través de la aplicación sistemática de prácticas lean (Agudo et al. 2013). Belekoukias et al. (2014) sugiere que los métodos y herramientas de lean ayudan a las organizaciones manufactureras a mejorar sus operaciones y procesos. Sin embargo, el efecto real de estos métodos y herramientas sobre las medidas contemporáneas de rendimiento operacional, es decir, costo, velocidad, fiabilidad, calidad y flexibilidad, aún no está claro.

Mediante el análisis de la carga de trabajo se determinan los recursos necesarios y el esfuerzo para llevar a cabo las diferentes operaciones, identificando las necesidades reales que se presentan para el recurso humano, definiendo necesidades de modificación en la asignación laboral con miras a la consecución de las metas establecidas (Becerra et al., 2016). Por lo tanto, si se conoce la cantidad de pedido y el ciclo de entrega, no es difícil calcular el plan de trabajo para un día, el gerente de la planta debe ser capaz de averiguar el tamaño mínimo del grupo de trabajadores bajo la condición previa de cumplir la orden a tiempo (Feng \& Fan, 2014).

Para Ortiz \& Caicedo (2015), identificar las restricciones del sistema productivo para el desarrollo de un modelo programación, determina las cantidades óptimas de fabricación, maximizando el throughput (utilidades) para un período de tiempo dado, la teoría de restricciones (TOC) se centra en el papel que juegan las restricciones en los sistemas con el fin de mejorar el desempeño del mismo hacia la meta. Las restricciones pueden ser de recursos internos, de mercado y de políticas.

Mediante la implementación de las metodologías propuestas por TOC, se logró un mayor nivel de sincronización en los procesos logísticos de la cadena de suministro de una empresa, programar la producción influye sustancialmente en la aplicación de la Teoría de Restricciones, ya que si se trabaja con un sistema de producción sobre pedido es mucho más fácil realizar la planeación de producción, controlar el flujo del producto, identificar el recurso cuello de botella y organizar los amortiguadores de existencias porque se programa para cumplir con una fecha de entrega; (Marín \& Gutiérrez, 2013).

Las prácticas Just in time (JIT) son muy útiles para mejorar el rendimiento operacional, estas prácticas, que representan el núcleo de la metodología de gestión Lean, fueron desarrolladas en primer lugar en Toyota, donde la producción es altamente repetitiva y durante muchos años los investigadores han pensado que esta metodología podría aplicarse en contextos caracterizados por sistemas de fabricación repetitivos solamente. Recientemente algunos autores han refutado este punto de vista, proporcionando evidencia empírica de que las prácticas JIT pueden ser implementadas con éxito también en contextos no repetitivos (Bortolotti et al., 2013). 
Reyes et al. (2016) señala que después de haber intercambiado ideas con expertos en materia de calzado sobre las deficiencias que afectan a los procesos industriales en las empresas locales, se evidencia que la industria del calzado en el Ecuador se encuentra inmersa en procesos de tecnificación cada vez más avanzados. Para Dos Santos et al. (2016), estimar la capacidad productiva disponible es determinante para satisfacer la demanda actual y futura del mercado. Los desajustes entre capacidad y demanda pueden dar lugar a dos situaciones no deseadas: la primera relaciona al aumento desmedido en la capacidad productiva, a veces para evitar nuevos entrantes en el mercado; la segunda se da cuando se dispone de muy poca capacidad de producción, perdiendo oportunidades en el mercado haciendo que los clientes se sienten insatisfechos. Por lo tanto, la inversión en capacidad debe planificarse cuidadosamente.

La correcta selección de la herramienta de planificación o control pueden ser de gran utilidad para las organizaciones y contribuir a incrementar los indicadores de productivos y elevar el servicio que las mismas brindan al cliente (Tamayo \& Urquiola, 2014).

Programar la producción comprende fases o pasos sistemáticos que permiten generar cargas de trabajo uniformes administrando correctamente los recursos de la empresa, Hu et al. (2016) ha indicado que, en la fase de planificación se toma la decisión relativa al período regular de disponibilidad de recursos con el fin de minimizar los costos de recursos esperados. En la fase de ejecución, se presentan y examinan un método de reparación de órdenes programadas para la reprogramación junto con dos procedimientos de asignación de recursos reactivos como la acción correctiva cuando los retrasos están más allá de un cierto umbral de amortiguación para exhibir un sistema de control de costes.

La investigación se la realiza en la industria manufacturera de calzado de la provincia de Tungurahua-Ecuador, específicamente en el proceso de troquelado de Cuero, se consideraron dos actividades dentro del proceso de troquelado, estas corresponden al corte de cuero y al corte de suplementos (forros, telas, esponjas, plantillas de celfil).

El objetivo de esta investigación es implementar un sistema de producción sobre pedido que controle el flujo del material, identifique el recurso cuello de botella y sincronice la manufactura del calzado para cumplir con la fecha de entrega. El modelo propuesto busca proporcionar una referencia para una gestión óptima y eficiente de la materia prima, utilizando la teoría de las restricciones y herramientas de manufactura esbelta, de esta manera se distribuirá correctamente los recursos de la empresa. La ejecución de este modelo plantea elevar los niveles eficiencia del proceso, marcando un aumento en la productividad de la empresa, de esta forma se busca aumentar las ventajas competitivas del sector manufacturero de calzado.

Para cumplir dicho objetivo la investigación se vale de herramientas Lean Manufacturing y la determinación de tiempos de producción, capacidades de producción y cuellos de botella. Determinar la capacidad de producción permite precisar el número de cortes de cuero y complementos que se pueden producir en una unidad de tiempo determinado. El tiempo estándar es elemental para realizar el cálculo de la capacidad en cada línea de producción. Reyes (2014), emplea una ecuación que divide la unidad para el tiempo estándar con el fin de determinar la capacidad de producción de cada modelo de calzado. Se toma los valores correspondientes a las capacidades de cada modelo de calzado y se la multiplica por el número de células de manufactura determinando en el análisis del recurso humano que se realiza para cada empresa, de esta manera se distribuye la cantidad de pares de calzado requeridos entre las células de manufactura haciendo más rápido el flujo del proceso.

Gundogar et al. (2016), recomienda encontrar los cuellos de botella en la línea de producción para equilibrar el flujo de material semi acabado. Estos cuellos de botella son investigados y varios escenarios diferentes son probados para mejorar el sistema de fabricación actual. 
El problema con un tema principal basado en la eliminación del cuello de botella se resuelve utilizando la teoría de Goldratt y Cox.

Para Reyes et al. (2017), no calcular la capacidad de producción real interfiere con los procesos de manufactura fabricación desaprovechando el tiempo de producción y la cantidad de recursos humanos destinados.

\section{MATERIAL Y MÉTODOS}

Para el desarrollo del modelo de programación del proceso de manufactura de calzado se determinó una muestra de 4 modelos de calzado, de una población de 104 según registros de la Cámara Nacional de Calzado (CALTU). Los modelos seleccionados, se manufacturaron en líneas de producción empleando métodos de corte diferentes para cada modelo: calzado casual de hombre, deportivo de mujer y seguridad industrial.

\section{Análisis de las Operaciones}

Se observó que la distribución del área de troquelado está dada por células de trabajo, el proceso inicia con la selección de bandas de cuero de cuero hasta la colocación de los cortes en gavetas para ser transportados hacia el siguiente proceso productivo como se observa en la en la Figura 1. La manufactura en el proceso de troquelado se realiza de forma empírica, no se pronostica volumen de producción, existe además una mala distribución del recurso humano y maquinaria.

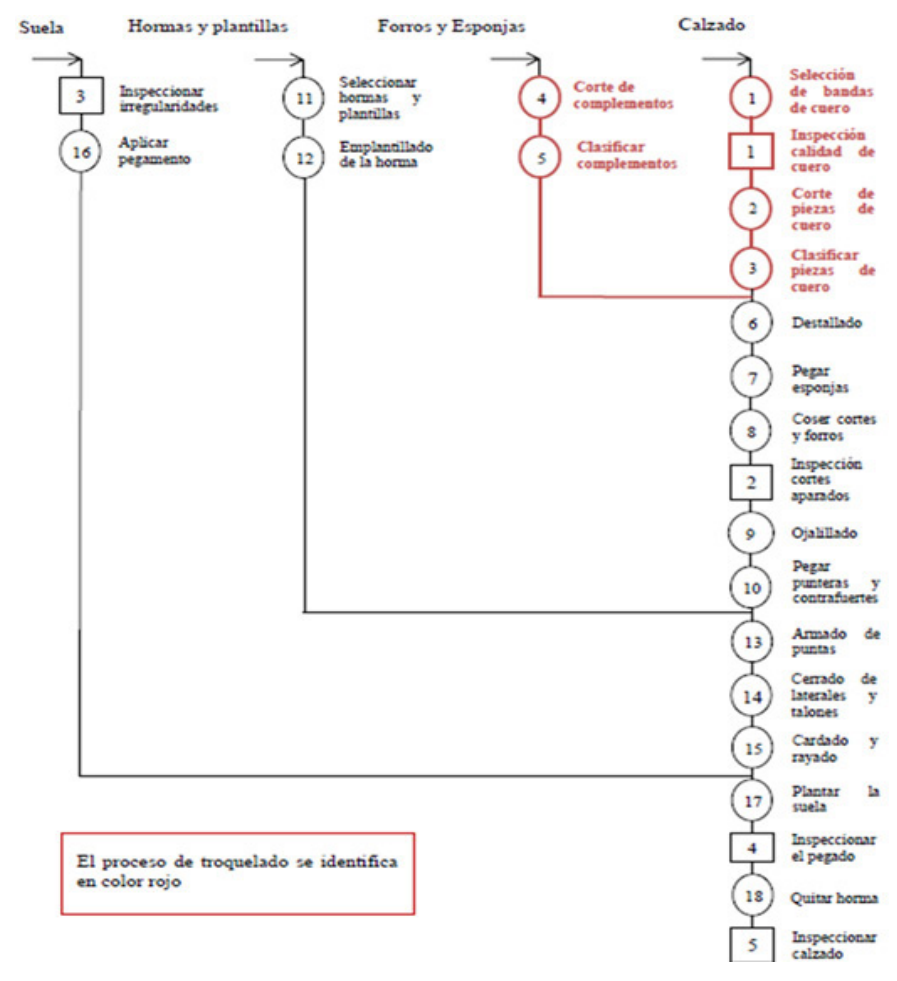

Figura 1. Diagrama de Ensamble de Calzado 


\section{Estudio de la Demanda}

Para analizar el proceso de corte se realizó una clasificación de inventarios $A B C$, esto permite identificar los productos de mayor comercialización. Se estimó la demanda futura en base al histórico de ventas de dos periodos anteriores de cada empresa, de acuerdo a la variación de la demanda de los modelos de estudio. El modelo de pronóstico corresponde a un modelo cuantitativo de series de estacionales, debido a las fluctuaciones que se dan de mes a mes como se muestra en las Figuras 2,3 y 4 . El método de pronóstico corresponde a regresión lineal.

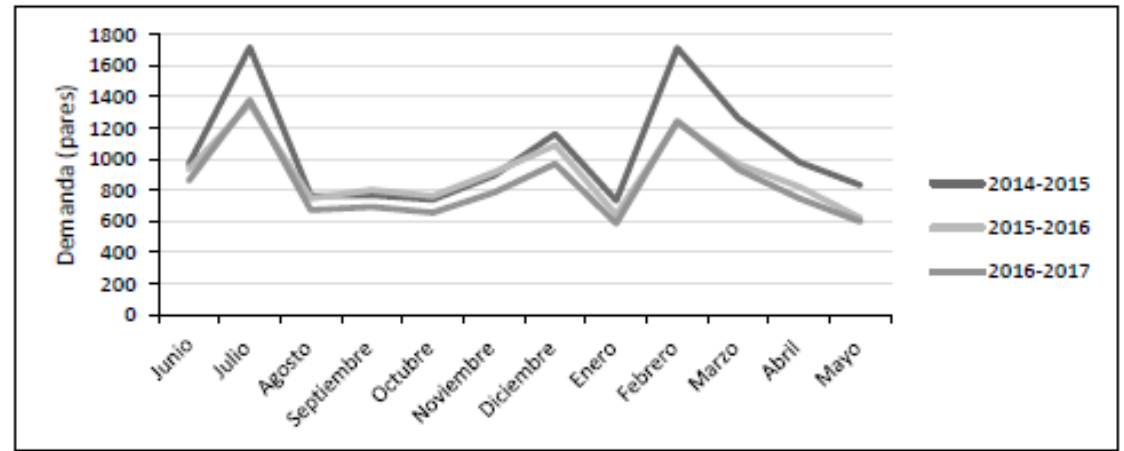

Figura 2. Fluctuaciones de Tres Periodos. Modelo Seguridad Industrial

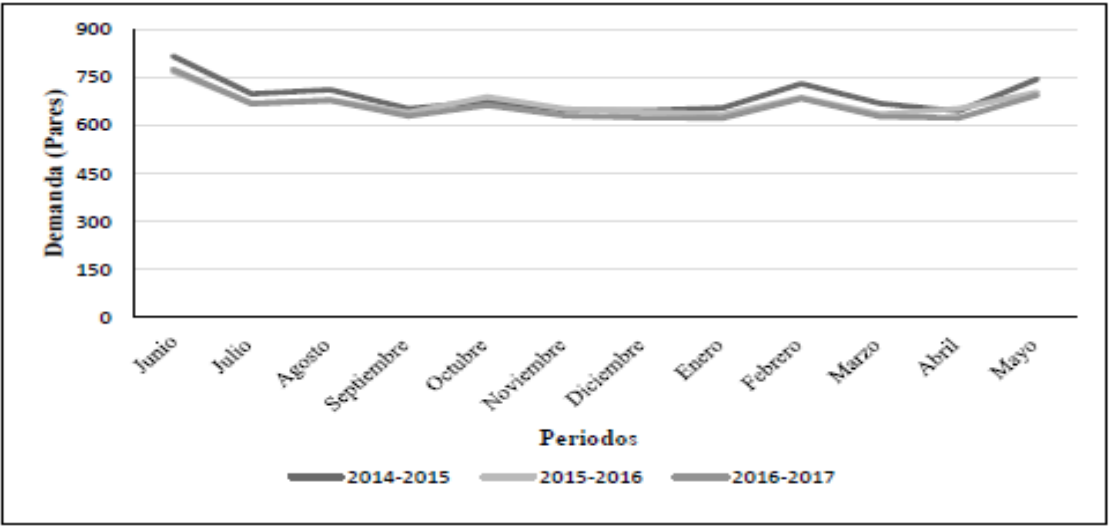

Figura 3. Fluctuaciones de Tres Periodos. Modelo Seguridad Industrial 


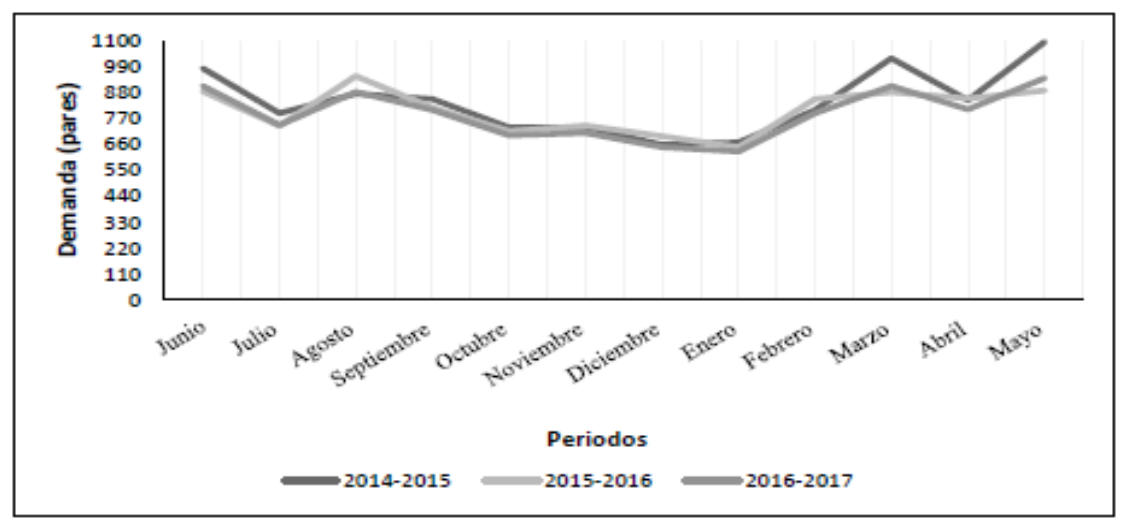

Figura 4. Fluctuaciones de Tres Periodos. Modelo Deportivo

Se consideró la demanda pronosticada para un año, desde los meses de junio del 2016 a mayo del 2017. No se requiere incrementar la demanda que viene dada desde el proceso de montaje, se toma esta demanda como la orden de producción para el proceso de troquelado, de esta manera se implementa el sistema de producción Pull para eliminar cualquier costo relacionado a mantener inventario.

En la Tabla 1 se resume la demanda mensual para cada modelo de estudio.

Tabla 1. Demanda Mensual

\begin{tabular}{|c|c|c|c|c|}
\hline Modelo & Casual & Deportivo & Seguridad & $\begin{array}{c}\text { Seguridad } \\
\text { Inyección }\end{array}$ \\
\hline jun-16 & 236,0 & 570,0 & 659,0 & 877,0 \\
\hline jul-16 & 131,0 & 402,0 & 1216,0 & 1402,0 \\
\hline ago-16 & 111,0 & 436,0 & 374,0 & 618,0 \\
\hline sep-16 & 102,0 & 518,0 & 545,0 & 689,0 \\
\hline oct-16 & 118,0 & 332,0 & 430,0 & 637,0 \\
\hline nov-16 & 93,0 & 366,0 & 590,0 & 801,0 \\
\hline dic-16 & 63,0 & 217,0 & 559,0 & 985,0 \\
\hline ene-17 & 110,0 & 357,0 & 487,0 & 501,0 \\
\hline feb-17 & 132,0 & 415,0 & 970,0 & 1386,0 \\
\hline mar-17 & 89,0 & 559,0 & 718,0 & 963,0 \\
\hline abr-17 & 81,0 & 436,0 & 508,0 & 710,0 \\
\hline may-17 & 140,0 & 559,0 & 364,0 & 509,0 \\
\hline
\end{tabular}

\section{Análisis de la Capacidad de Producción}

Para calcular la capacidad de producción se utilizó la Ecuación 1 (Reyes, 2014) capacidad de producción $=\frac{1}{T_{S}}$ 
Donde Ts es el tiempo estándar, el mismo que se obtuvo de un estudio de tiempos considerando un lote de producción.

En la Tabla 2 se muestra las capacidades de cada línea de producción.

Tabla 2. Capacidad de Producción

\begin{tabular}{|c|c|c|c|}
\hline \multirow{2}{*}{ Modelo } & Material Cortado & $\begin{array}{c}\text { Capacidad } \\
\text { Producción (parl } \\
\text { hora) }\end{array}$ & $\begin{array}{c}\text { Capacidad } \\
\text { Producción } \\
\text { (par/día) }\end{array}$ \\
\hline \multirow{2}{*}{ Casual Hombre } & Cuero & 11.13 & 89.1 \\
\cline { 2 - 4 } & Complementos & 22.22 & 177.8 \\
\hline \multirow{2}{*}{ Deportivo Mujer } & Cuero & 24.19 & 193.5 \\
\cline { 2 - 4 } & Complementos & 30.15 & 241.2 \\
\hline \multirow{2}{*}{\begin{tabular}{c} 
Seguridad Industrial \\
\cline { 2 - 4 }
\end{tabular}} & Cuero & 21.13 & 169.0 \\
\hline \multirow{2}{*}{$\begin{array}{c}\text { Seguridad Industrial } \\
\text { Inyección }\end{array}$} & Complementos & 37.50 & 300.0 \\
\cline { 2 - 4 } & Complementos & 21.13 & 169.0 \\
\hline
\end{tabular}

\section{Cuello de Botella del Proceso de Troquelado}

Se determinaron los cuellos de botella para determinar el ritmo de trabajo del proceso de menor capacidad de producción, la misma que limita el flujo del proceso en las líneas, las mismas que se expresan en la Tabla 3 .

Tabla 3. Capacidad Mínima de Producción

\begin{tabular}{|c|c|c|c|}
\hline Modelo & $\begin{array}{c}\text { Capacidad de } \\
\text { producción Corte } \\
\text { de Cuero (pares/ } \\
\text { día) }\end{array}$ & $\begin{array}{c}\text { Capacidad de } \\
\text { producción Corte de } \\
\text { Complementos (pares/ } \\
\text { día) }\end{array}$ & $\begin{array}{c}\text { Capacidad } \\
\text { Mínima (pares/ } \\
\text { día) }\end{array}$ \\
\hline Casual & 89 & 178 & 89 \\
\hline Deportivo & 194 & 241 & 194 \\
\hline Seguridad & 169 & 300 & 169 \\
\hline Seguridad Inye. & 169 & 300 & 169 \\
\hline
\end{tabular}

A continuación se redacta el modelo de programación de producción planteado para el proceso de troquelado, en el que se considera capacidades de producción y cuellos de botella calculados, para ello se consideraron los siguientes pasos:

\section{Paso 1: Implementar la Teoría de Restricciones (TOC)}

De igual manera que en el modelo propuesto para todo el sistema de producción, para el proceso de troquelado se desarrollarán 3 de los 5 pasos expuestos en la metodología TOC, el cuello de botella se determinará en base al cálculo de la capacidad mínima de cada línea de 
producción. Se empleará además la herramienta lógica TAC (tambor, cuerda y amortiguador) de la teoría de restricciones para determinar cada uno de estos componentes.

1. Identificar las Restricciones: Cuando un proceso no fluye continuamente y genera tiempos de espera prolongados es porque dentro de él existen restricciones que limitan la producción. La restricción de la planeación de la producción se considera como la que genera mayor conflicto en el proceso de troquelado al no considerar las capacidades mínimas de producción de la cada línea de producción. Otra limitación a considerar es la restricción del recurso humano, yaqué determinar de la cantidad necesaria del recurso humano haría el proceso responda con mayor rapidez cumpliendo en menor tiempo las ordenes de producción. En la Figura 5 se muestra las restricciones identificadas de acuerdo al grado de importancia

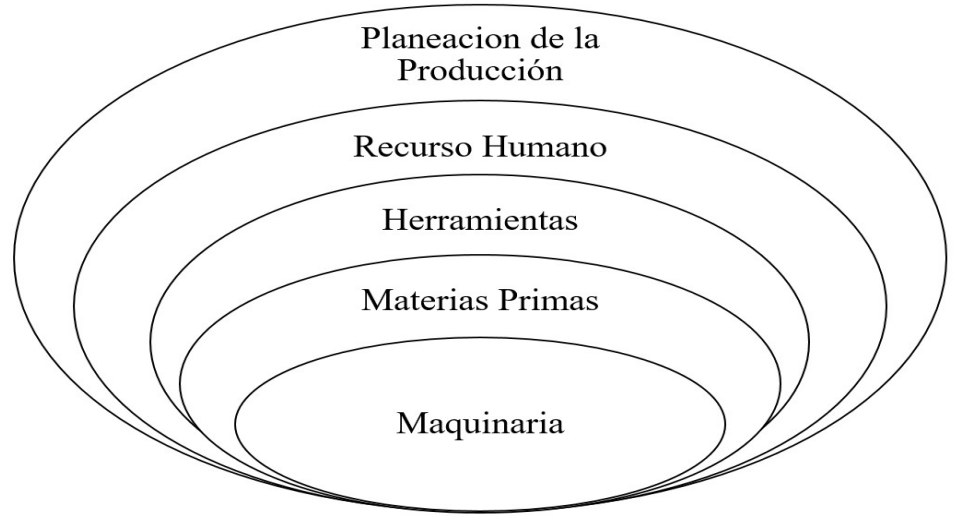

Figura 5. Restricciones del Proceso de Troquelado

2. Explotar las Restricciones: Desarrollar un modelo de planeación y programación para el proceso de troquelado, considerando la capacidad mínima de producción, hará que el proceso de manufactura sea flexible, determinando tiempos de producción para los modelos analizados en cada línea de producción. El tiempo restante de la jornada de trabajo será asignado para producir los otros modelos de calzado de la empresa. Maximizar el desempeño del recurso humano empleando células de manufactura para cada línea de producción, permitirá disminuir los tiempos de manufactura, de esta manera se controla el cuello de botella limitado por la capacidad de producción de cada modelo de calzado.

3. Subordinar las Restricciones: El modelo propuesto está subordinado al cuello de botella. Utilizando el fundamento TAC (Tambor, Amortiguador, Cuerda) de la Teoría de Restricciones se identifica cada elemento que permite subordinar al modelo.

El tambor es elemento que marca el ritmo de producción en este caso es el cuello de botella generado por la capacidad de producción mínima de cada línea de producción. Con la cuerda se contrala el cuello de botella atando el tambor del proceso al modelo de programación de la producción propuesto de esta manera se relaciona la capacidad de producción mínima con la variación de la demanda de calzado. Esta relación permite asignar el recurso humano necesario para cumplir con la demanda de acuerdo a la capacidad instalada y las células de manufactura estimadas en cada línea de producción siendo estos parámetros el amortiguador del modelo propuesto, el amortiguador ayuda al modelo a no descompensarse por las variaciones de la demanda. En la Figura 6 se muestra la interacción de estos elementos mencionados. 


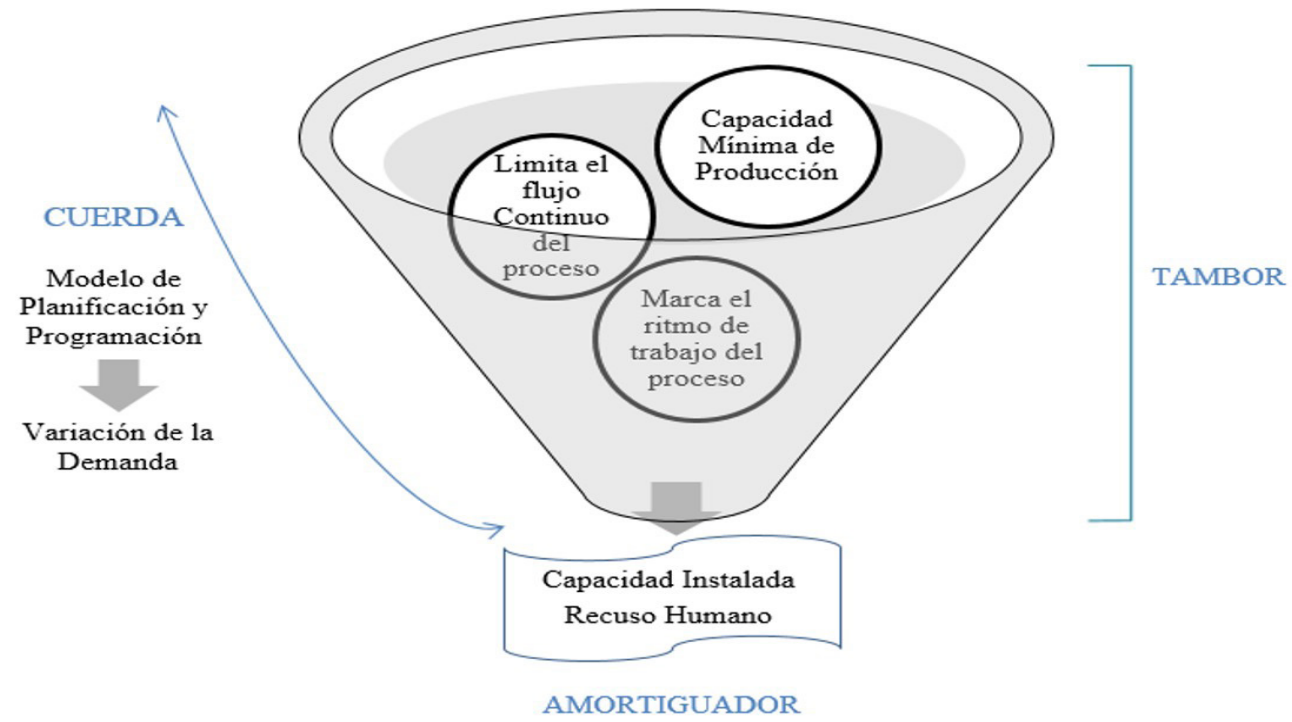

Figura 6. Tambor-Amortiguador-Cuerda del Modelo propuesto

\section{Paso 2: Secuenciación de Órdenes de Producción}

La coordinación de las actividades de producción y suministro de materiales podría ser útil para darle al gerente de operaciones una estimación del impacto de las decisiones de planificación teniendo en cuenta la interrelación entre las actividades de producción y suministro (Manzini \& Urgo, 2018). Implementado el sistema de producción Pull, se logra la correcta secuenciación de los procesos por medio de órdenes de producción, estas vienen dadas desde el proceso de montaje y son extendidas para los procesos de troquelado y aparado. Para ello es necesario implementar un sistema de producción tipo Pull y una serie de parámetros de producción expuesta a continuación.

Sistema de Producción Pull: Es una herramienta Lean Manufacturing, que permite optimizar el proceso productivo. Emplea la estrategia de fabricación por pedido, ya que esta estrategia satisface las prioridades de los procesos anteriores, Rusli (2015), menciona que los principales factores que influyen en la selección del sistema de producción Pull son la capacidad de cambio y la variedad de número de pieza a producir. Este tipo de sistema de manufactura, hace que el proceso de montaje jale del proceso de aparado los cortes unidos mediante costura, consecuentemente el proceso de aparado jala los cortes de cuero y complementos realizados en el proceso de troquelado. Para ello es necesario generar órdenes de producción donde se indique la demanda pronosticada para cada mes.

Orden de Producción: En la orden de producción se determina el número de pares a cortar por cada modelo de estudio, demás se identifican las tallas requeridas por modelo, a partir de ella se planifica la ejecución de las actividades que corresponden al proceso de Troquelado tomando en cuenta los recursos necesarios para llevar a cabo las operaciones de corte. Para la el modelo de planificación y programación propuesto, se establece que la orden de producción llegue cada inicio de semana. En la Figura 7 se muestra una orden de producción. 


\begin{tabular}{|c|c|c|c|c|c|c|c|c|c|c|c|c|c|}
\hline MES & \multicolumn{13}{|l|}{ JUNIO } \\
\hline SEMANA & \multicolumn{13}{|l|}{1} \\
\hline \multirow{2}{*}{ CÓDIGO } & \multirow{2}{*}{ MODELO } & \multicolumn{11}{|c|}{ TALLAS } & TOTAL \\
\hline & & 34 & 35 & 36 & 37 & 38 & 39 & 40 & 41 & 42 & 43 & 44 & \\
\hline 00CA873 & CASUAL & 0 & 0 & 0 & 2 & 7 & 8 & 7 & 5 & 2 & 1 & 0 & 32 \\
\hline 00SK568 & $\begin{array}{l}\text { DEPORTIVO } \\
\text { MUJER }\end{array}$ & 0 & 11 & 25 & 23 & 14 & 3 & 2 & 0 & 0 & 0 & 0 & 78 \\
\hline S09 & $\begin{array}{l}\text { SEGURIDAD } \\
\text { INDUSTRIAL }\end{array}$ & 0 & 0 & 0 & 1 & 20 & 24 & 24 & 11 & 5 & 4 & 0 & 90 \\
\hline S09-I & $\begin{array}{l}\text { SEGURIDAD } \\
\text { INYECCIÓN }\end{array}$ & 0 & 0 & 0 & 1 & 37 & 40 & 23 & 11 & 5 & 4 & 0 & 120 \\
\hline
\end{tabular}

Figura 7. Orden de Producción

Capacidad Diaria: Se toma los valores correspondientes a las capacidades de cada línea de producción y se la multiplica por el número de células de manufactura determinado en el análisis del recurso humano calculado para cada mes y por línea de producción, de esta manera se distribuye la cantidad de pares de calzado requeridos entre las células de manufactura haciendo más rápido el flujo del proceso. El número de las células de manufactura varían de acuerdo al mes debido a la estacionalidad de la demanda.

Capacidad Semanal: Se obtiene multiplicando la capacidad diaria de producción por el número de días laborables en la semana, de esta manera se conoce la capacidad máxima de producción por semana.

Turnos: Corresponden al número de turnos necesarios para cumplir con el requerimiento de producción, de acuerdo a la realidad de las pequeñas y medianas empresas se considera un turno diario de trabajo de ocho horas al día. Para calcular el número de turnos se emplea una relación matemática entre la capacidad semanal con la demanda establecida de donde se obtiene la ecuación 2 que permite determinar los turnos necesarios para cumplir con la demanda.

$$
\text { Turnos }=\frac{\text { Demanda Semanal }}{\text { Capacidad Semanal }} x \text { días de la semana }
$$

Para la Ecuación 2 (Turnos), la demanda semanal corresponde a cantidad a producir en una determina semana. La capacidad semanal es el número de pares que se producen por semana considerando. Como ejemplo para determinar los turnos se toma los parámetros de producción de la primera semana del mes de junio del modelo de casual.

$$
\begin{aligned}
& \text { Turnos }=\frac{\text { Demanda Semanal }}{\text { Capacidad Semanal }} \times \text { dias de la semana } \\
& \text { Turnos }=\frac{\text { Demanda Semanal junio semana } 1}{\text { Capacidad Semanal junio semana } 1} \times 3 \text { dias } \\
& \text { Turnos junio semana } 1=\frac{106 \frac{\text { pares }}{\text { semana }}}{534 \frac{\text { pares }}{\text { semana }}} \\
& \text { Turnos junio semana } 1=0,60 \text { dias }
\end{aligned}
$$

Días de Producción: Para programar la producción se debe definir las horas de producción 
que se necesita para cumplir con la demanda dada; de esta manera se sincronizan los procesos, estableciendo plazos determinadas de entrega. La cantidad de pares producidos por línea de producción.

\section{Paso 3: Estimar el cálculo del Recurso Humano y de las Células de Manufactura necesarias}

El enfoque de celdas de manufactura reduce los costos asociados al manejo de material lo que representa un ahorro anual, (Herrera \& Torres, 2016). Se asignará el personal necesario a cada línea de producción para cumplir con la orden de producción, con esto se logra optimizar el uso del recurso humano. Para la correcta asignación del recurso humano se debe considerar dos aspectos fundamentales para el desarrollo del modelo propuesto, el primero determinar la cantidad necesaria de células de manufactura para satisfacer la demanda dada desde el proceso de montaje, el segundo es estimar el número de personas que serán desinadas a cada célula de manufactura determinada para cada línea de producción.

\section{Calculo del Número de Células de Manufactura:}

La formación de las celdas se basa en la agrupación de partes y máquinas a partir de las similitudes entre estas. Las celdas de manufactura pueden asimilarse a pequeñas mini fábricas al interior de la planta donde se fabrican un grupo de piezas similares (Moncayo et al., 2014)

Cada línea de producción cuenta con un número determinado de células de manufactura, estas células se emplean para producir la demanda de los modelos que se manufacturan en cada línea de producción. Para disminuir los tiempos de producción del proceso de troquelado, se distribuye la demanda de un modelo de calzado analizado para un determinado número de células; estas células se encargarán de manufacturar la demanda correspondiente del modelo

Con los valores del mes de junio se plantea la relación para determinar el número de celdas considerando 6 células de manufactura disponibles.

Relación: $\quad$ Si 6928 ParesMes $\longrightarrow 6$ células de manufactura

1684 ParesMes $\longrightarrow$ x células de manufactura

De la relación planteada se obtiene la Ecuación 3.

$N^{\circ}$ Células $=\frac{(\text { Demanda Mensual Modelo estudiado }) x(\text { Célula determinadas })}{\text { Capacidad Semanal }}$

La Ecuación 3 ( $\mathrm{N}^{\circ}$ Células) relaciona la demanda mensual del modelo de estudio multiplicado por el número de células determinado para cada línea de producción, con la demanda total considerando todos los modelos de la línea de producción.

Cálculo del Número de Personas para las Células de Manufactura: Para determinar el número de personas por cada célula de manufactura se deben analizar las actividades de corte de cuero y corte de complementos. Para calcular el número de personas que conformaran la célula de manufactura, se relaciona la capacidad diaria de producción de cada modelo de calzado con la capacidad mínima de producción de la línea de producción, el número de personas se obtendrá sumando el resultado obtenido en cada línea de producción. De la relación planteada anteriormente se obtiene la Ecuación 4 mediante la cual se determina el 
cálculo de la siguiente forma:

$$
N^{\circ} \text { de personas }=\frac{\text { Capacidad Mínima de Producción }}{\text { Capacidad de Producción }}
$$

La Ecuación 4 ( $N^{\circ}$ Personas) relaciona la capacidad mínima de producción y la capacidad total de producción. Tomando de ejemplo al modelo casual se determina el número de personas necesarias por célula de manufactura, considerando que la capacidad mínima de producción para este modelo de calzado es de 89 pares por día de trabajo.

Proceso de Corte de Complementos:

$$
N^{\circ} \text { de Personas }=\frac{89,05 \frac{\text { pares }}{\text { día }}}{177,78 \frac{\text { pares }}{\text { día }}} \times 0,5
$$

\section{RESULTADOS Y DISCUSIÓN}

La demanda de calzado es estacional, los intereses de compra de los consumidores suben en los meses de febrero y junio, en la Figura 8 se determina que los modelos de seguridad industrial son los de mayor demanda en estos meses, estos valores bordean a los 1400 pares en los meses mencionado anteriormente. La demanda del resto de modelos se mantiene estable a lo largo del año, teniendo valores de producción por debajo de los 800 pares.

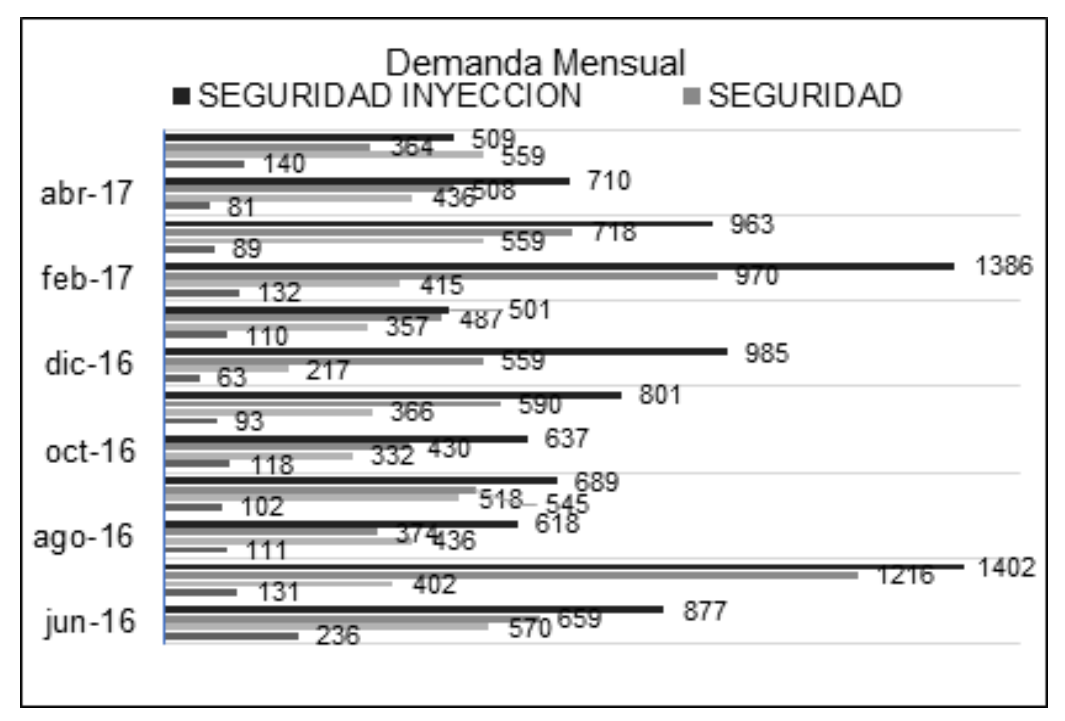

Figura 8. Demanda Mensual de Calzado

En la Figura 9, se muestran los porcentajes de utilización del operador y la maquinaria, para ello se relacionando el tiempo manual y el tiempo de máquina. 


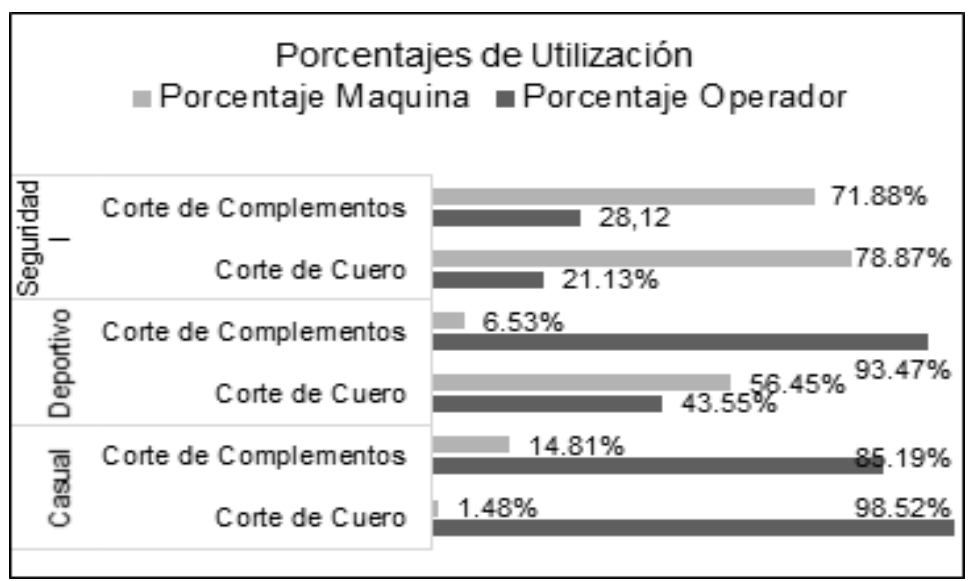

Figura 9. Porcentajes de Utilización de Maquinaria y Operación

En la Tabla 4 se muestra las horas equivalentes al porcentaje de utilización de la máquina y el operador en base a la capacidad de producción por modelo en una jornada de trabajo. El número de personas determinado para las células de manufactura en cada línea de producción es dos, de esta manera se puede distribuir el trabajo de corte y complementos para cada uno de las personas por separado, es decir que el proceso de corte de cuero y el proceso de corte de complementos serán ejecutados paralelamente.

Tabla 4. Tiempo Equivalente al Porcentaje de Utilización

\begin{tabular}{|c|c|c|c|}
\hline Modelo & Actividad & $\begin{array}{c}\text { Tiempo Manual } \\
\text { (horas) }\end{array}$ & $\begin{array}{c}\text { Tiempo de Máquina } \\
\text { (horas) }\end{array}$ \\
\hline \multirow{2}{*}{ Casual } & Corte de Cuero & 7,9 & 0,1 \\
\cline { 2 - 4 } & Corte de Complementos & 6,8 & 1,2 \\
\hline \multirow{2}{*}{ Deportivo } & Corte de Cuero & 3,4 & 4,6 \\
\cline { 2 - 4 } & Corte de Complementos & 7,5 & 0,5 \\
\hline \multirow{2}{*}{$\begin{array}{c}\text { Seguridad } \\
\text { Industrial }\end{array}$} & Corte de Cuero & 1,7 & 6,3 \\
\cline { 2 - 4 } & Corte de Complementos & 2,2 & 5,8 \\
\hline
\end{tabular}

En la Figura 10 se indica la capacidad de la maquinaria utilizada en cada línea de producción. 


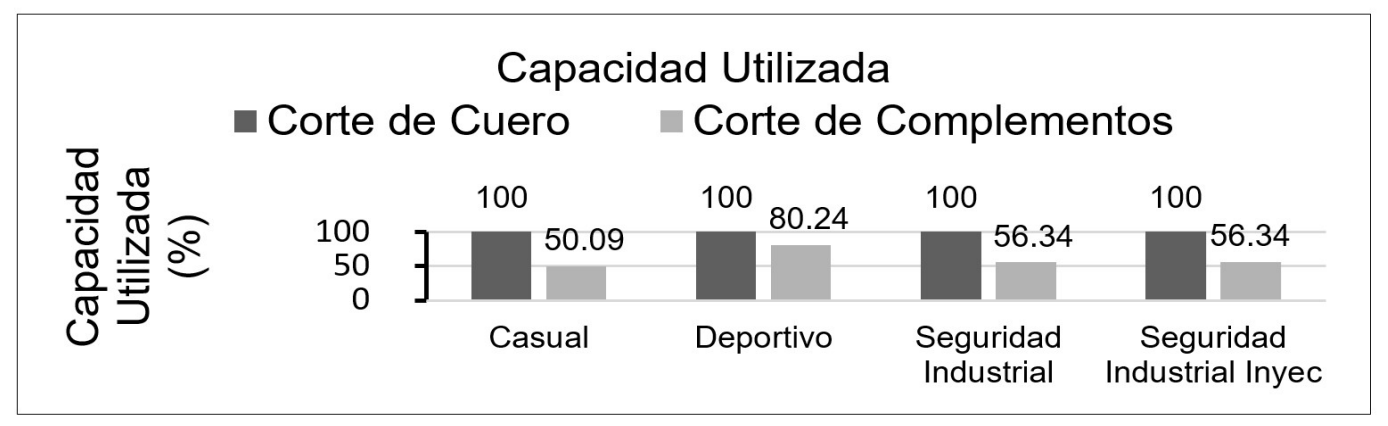

Figura 10. Porcentajes de Utilización de la Capacidad Instalada

Se consideran dos escenarios para la experimentación del modelo de programación de la producción propuesto en el proceso de troquelado, el primero corresponde a la situación actual de la empresa en donde se consideran volúmenes de producción establecidos por la empresa y el segundo escenario corresponde a los parámetros calculados en base al modelo de planeación propuesto, considerando las restricciones del proceso productivo. Para el cálculo de la eficiencia se relaciona los valores de la capacidad programada y la capacidad efectiva. En la Figura 11 se determina el aumento de la eficiencia en la manufactura de cada modelo.

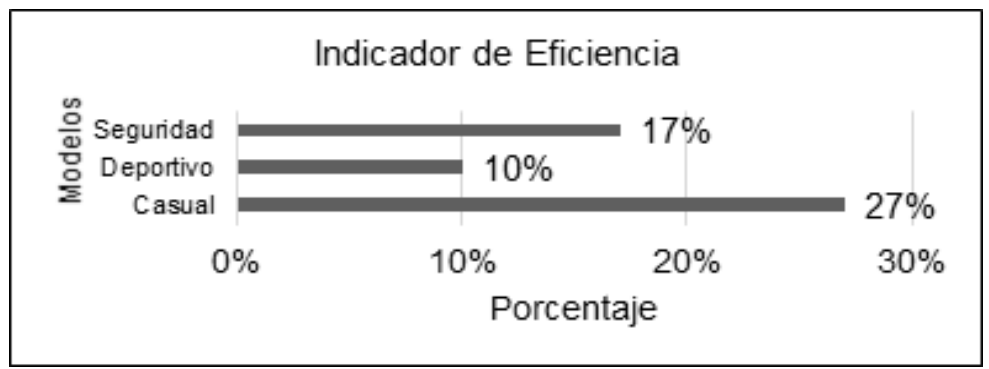

Figura 11. Indicadores de Eficiencia

\section{CONCLUSIONES}

Identificar las restricciones del proceso de troquelado influye sustancialmente en el modelo de programación de la producción, aplicando la Teoría de la Restricciones se identifica en base al cálculo de la capacidades de producción, el recurso cuello de botella o tambor el cual marca el ritmo de producción, para organizar y distribuir los amortiguadores recurso humano y capacidad instalada.

En el modelo propuesto, se determinó a la cuerda como el elemento que comunica la demanda con el proceso restringido liberando la producción al ritmo de la capacidad mínima de manufactura de cada línea de producción, de esta manera se programa para cumplir con una fecha de entrega establecidas en el modelo propuesto. 
En función a la capacidad mínima de producción de cada modelo analizado, se determina el porcentaje de utilización de la maquinaria empleada en la manufactura de cada modelo. El porcentaje de utilización de la maquinaria en la actividad de corte de cuero corresponde al $100 \%$, esto significa que la maquinaria se la aprovecha en su totalidad durante todo el tiempo que se toma en manufacturar la demanda; los porcentajes de utilización de la maquinaria en el proceso de corte de complementos varían entre un $50,1 \%$ hasta $80,2 \%$ de utilización debido a que la capacidad de producción de este proceso supera a la capacidad de producción del corte de cuero, el restante de capacidad es empleado para producir otros modelos de la empresa.

Con la aplicación del sistema Pull se reduce los tiempos muertos entre proceso mediante el cálculo de los turnos y el tiempo de producción de cada modelo de calzado estudiado, ya que se utilizó la estrategia de producción bajo pedido permitiendo así sincronizar los procesos de manufactura de calzado, ya que este sistema genera una dependencia entre procesos haciendo que la demanda se ejecute en el menor tiempo posible para aprovechar la jornada laboral.

El modelo propuesto facilita el flujo de los procesos mediante el enfoque JIT, para ello se generan ordenes de producción que se ajustan en todo momento a la variación de la demanda, a partir de ellas se planifica la ejecución de las actividades que corresponden al proceso de Troquelado yaqué nada se producirá hasta que realmente se requiera.

La distribución eficiente de los recursos permitió la reducción de los tiempos de proceso generando cargas uniformes de trabajo, de esta manera cada línea de producción trabajara en forma paralela, aumentando la eficiencia de la capacidad de producción en valores comprendidos desde el $10 \%$ hasta el $27 \%$.

\section{REFERENCIAS}

AGUDO, S., ÁLVAREZ, R., and DOMINGO, R., Modelo de mejoras eficientes y sostenibles en un sistema de producción esbelto a través de procesos de innovación ambiental. Journal of Cleaner Production, 2013,47,141-148.

BECERRA, M., AYALA, S., ASTROS, J., and GONZÁLEZ, E. Algoritmo para el cálculo de cargas de trabajo. Revista Ingeniería Industrial, 2016,15(1),5346-5366.

BELEKOUKIAS, I., GARZA, J., and KUMAR, V. The impact of lean methods and tools on the operational performance of manufacturing organisations. International Journal of Production Research, 2014, 52(18),5346-5366.

BORTOLOTTI, T., DANESE, P., and ROMANO, P. Assessing the impact of just-in-time on operational performance at varying degrees of repetitiveness. International Journal of Production Research, 2013, 51(4),117-1130. [Fecha consulta: 15 de Mayo de 2017] Disponible en: <http://www.tandfonline.com/doi/abs/10.1080/00207543.2012.678403> 
DOS SANTOS, L., MAYERLE, S., DOS SANTOS, C., and MENDES, M. Industrial capacity planning in oligopolistic markets. Springer Optimization and Engineerin, 2016, 17(4), 761780. [Fecha consulta: 10 de Mayo de 2017] Disponible en: <https://link.springer.com/ article/10.1007/s11081-016-9318-1>

FENG, Y., and FAN W. A hybrid simulation approach to dynamic multi-skilled workforce planning of production line. In: TOLK, Andreas., DIALLO, Saikuo., RYZHOV, Ilya., YILMAZ, Levent., BUCKLEY, Stephen., and MILLER, Jonh. Proceedings of the 2014 Winter Simulation Conference. Institute of Electrical and Electronics Engineers. IEEE,2014, 1664-1675.

GUNDOGAR, E., SARI, M., and KOKCAM, A. Dynamic bottleneck elimination in mattress manufacturing line using theory of constraints. Springer Plus, 2016,5(1), 1276. [Fecha consulta: 15 de Mayo de 2017] Disponible en: < https://link.springer.com/article/10.1186/ $\underline{\text { s40064-016-2947-1> }}$

HERRERA, F., and TORRES, J. Desarrollo e implementación de una metodología para el diseño de celdas de manufactura en pymes. Revista Latino-Americana de Inovação e Engenharia de Produção, 2016, 4(6), 20-34. [Fecha consulta: 10 de Abril de 2017] Disponible en: <http://revistas.ufpr.br/relainep/article/view/49746/29884>

HU, X., DEMEULEMEESTER, E., CUI, N., WANG, J., and TIAN W. Improved critical chain buffer management framework considering resource costs and schedule stability. Flexible Services and Manufacturing Journal, 2016,159-183. . [Fecha consulta: 10 de Abril de 2017] Disponible en: <https://link.springer.com/article/10.1007/s10696-016-9241-y>

MANZINI, M. and URGO, M. A risk based approach to support the supplying of components in a MTO assembly process. Journal of Manufacturing Systems, 2018, 46,67-78. . [Fecha consulta: 02 de Abril de 2017] Disponible en: <https://www.sciencedirect.com/science/article/ pii/S0278612517301474>

MONCAYO, C., GARZÓN, D., and ARROYO, J. Métodos discretos basados en quimiotaxis de bacterias y algoritmos genéticos para solucionar el problema de la distribución de planta en celdas de manufactura. Ciencia e Ingeniería Neogranadina, 2014, 24,(1),6-28. Disponible en: <https://revistas.unimilitar.edu.co/index.php/rcin/article/view/5/3>

ORTIZ, V. and CAICEDO, Á. Procedimiento para la programación y control de la producción de una pequeña empresa. Revista Ingeniería Industrial, 2015,14(1),89-104. .[Fecha consulta: 10 de Abril de 2017] Disponible en: <http://revistas.ubiobio.cl/index.php/RI/article/ view/1917/1833>

REYES,J., ALDAS, D., MORALES, L., and GARCÍA, M. Evaluación de la capacidad para montaje en la industria manufacturera de calzado. Ingeniera Industrial, 2016, 37(1),1423. [Fecha consulta: 10 de Febrero de 2017] Disponible en: <http://scielo.sld.cu/scielo. php?script=sci_arttext\&pid=S1815-59362016000100003> 
REYES, J., ALDAS,D., SALAZAR,E ., ARMENDARIZ, E., ALVAREZ, K., NUÑEZ J. and GARCIA, M. Finite Progressive Planning for the Assembly Process in Footwear. Materials Science and Engineering, IOP Conference Series, 2017,212,1-10. [Fecha consulta: 10 de Marzo de 2017] Disponible en: <http://iopscience.iop.org/article/10.1088/1757$\underline{899 X / 212 / 1 / 012020>}$

RUSLI, M., JAFFAR, A., KAYAT, S., ALI, M. Selection criterion of production methods used in the kanban pull system at Malaysian auto suppliers. In: Industrial Engineering and Operations Management (IEOM), 2015 International Conference on. IEEE, 2015. 1-5.

TAMAYO, A., and URQUIOLA, I. Concepción de un procedimiento para la planificación y control de la producción haciendo uso de herramientas matemáticas. Revista de Métodos Cuantitativos para la Economía y la Empresa, 2014,18,130-145. [Fecha consulta: 9 de Marzo de 2017] Disponible en: <http://www.redalyc.org/articulo.oa?id=233132797008> 
ISSN 0717-9103

revista Ingeniería Industrial-Año 16 №3, 2017

ISSN Online 0718-8307

Universidad del Bío-Bío 